\title{
The Interdisciplinary Challenge
}

LEROY HOOD

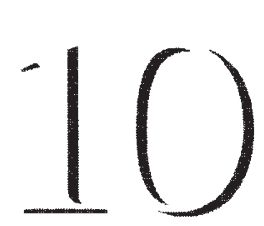

As we move into the 21 st century, biotechnology is changing profoundly. We are developing increasingly powerful tools for acquiring and manipulating biological information. In CELEBRATING living organisms, 3.7 billion A DE C A DE OF years of evolution have created E X C E L L E N C E a repository of one-dimensional genetic information that is ultimately translated into three-dimensional molecular machinery. Proteins and other biological macromolecules organize themselves into successively more complex entities - protein cascades, organelles, cells, tissues, and ultimately organisms. Many of life's basic properties arise from complex systems or networks of informational units.

In my view, biotechnology's central challenge is to develop tools and approaches for analyzing the complex systems and networks of living organisms. These analyses must include (1) delineating the elements of the network, (2) determining the connections between the elements, (3) forging computational tools for modeling these complex systems, and (4) the ability to test these computational hypotheses against the living reality. No such tools exist. Creating them will require powerful tools from other disciplines-applied mathematics, applied physics, bioengineering, chemistry, computer science, and engineering.

The human genome project, an effort to delineate the network of 50,000-100,000 genes governing human growth and development, illustrates the diverse challenges facing contemporary biotechnology. This endeavor has a two-fold objective: to develop automated, high-through-put, cost-effective tools and to decipher the genetic, physical, and sequence maps of the human and several model organism genomes (yeast, nematode, Drosophila, mouse). As the genomes unfold, biotechnology faces new opportunities and technical challenges:

- Can we identify the 50,000-100,000 human genes directly from DNA sequence data?

- Given knowledge of all gene sequences, how can we determine the functions of their corresponding proteins? Answering will require new technologies.

- Can computational methods identify gene control sequences? Will these molecular regulatory addresses help reveal how nature coordinates gene-network expression and function?

-Will we uncover an entire lexicon of protein motifs, protein building blocks from which we can infer protein structures as well as functions?

- How will we deal with information about polymorphisms associated with the predispositions to common diseases (cardiovascular, cancer, autoimmune, etc.)? Many of these will be polygenic, requiring new approaches to mapping and identifying individual genes. Developing and applying new therapies-to manipulate biological information and circumvent these predispositions-will present even greater challenges. Meeting them, though, will usher in an era of truly preventive medicine, sometime early in the $21 \mathrm{st}$ century.

To fulfill this promise, biotechnology will have to become more, not less, interdisciplinary, finding a way to promote collaborations with scientists outside biology. We will have to develop multidisciplinary centers committed to deciphering biology's complex systems and networks. Any such center will have to have certain characteristics and values:

- It must be committed to two tenets: First, that technology development should be driven by leading edge biology. And second, that the new tools, once developed, must be validated through application to important frontier problems of biology and medicine.

- It should draw its members from a wide variety of scientific backgrounds. These individuals will combine independent research efforts with a simultaneous commitment of their diverse skills to the joint development of complex technologies (e.g., large-scale DNA sequencing).

- The center should interact with other departmentsapplied mathematics, applied physics, bioengineering, chemistry, computer science, and electric engineering - to enliven biology with other leading edge technologies (despite the obstacles presented by differing disciplinary languages and presumptions), especially by students and young faculty.

-The center should be "vertically integrated" with a mix of scientists with expertise in protein chemistry, nucleic acid chemistry, molecular biology, biological computation, and biology.

Such centers would be well equipped for the sort of systems analysis and integration needed to develop critical complex technologies (e.g., the challenge of identifying the functions of genes).

Computation will play a critical role in biology and medicine of the future: biologists must become computer literate. At the same time, biological information systems may provide computer scientists with fundamental new insights.

The training of young scientists must become more interdisciplinary. Some Ph.D. programs may employ dual mentors - one biology department and a second in, say, computer science. A student might choose an important biological problem, create a new computational tool, and then apply the tool to discovery.

Most scientific disciplines of science pass through golden ages in which new approaches open the door to fundamental new knowledge. Biotechnology, the deciphering and use of biological information, is just beginning to move into its golden age. Just beginning.

\author{
Leroy Hood is \\ Gates Professor \\ and chairman of \\ the department \\ of molecular \\ biotechnology at \\ the University \\ of Washington \\ in Seattle. \\ The views \\ expressed \\ here are \\ the author's own \\ and not \\ necessarily \\ those of \\ Bio/Technology.
}

